\title{
Instrumento de Avaliação de Competências Aplicando a Lógica Paraconsistente
}

\section{Anotada Evidencial E $\tau$}

\author{
Skills Assessment Questionnaire Applying the Paraconsistent Annotated Evidential Logic E $\tau$ \\ Cuestionario de Evaluación de Habilidades que Aplica a Logica Paraconsistente Anotada
} Evidencial E $\tau$

\author{
Samira Sestari do Nascimento \\ ORCID: https://orcid.org/0000-0001-7429-6033 \\ Universidade Paulista, Brasil \\ E-mail: sestarisamira@gmail.com \\ Irenilza de Alencar Nääs \\ ORCID: https://orcid.org/0000-0003-0663-9377 \\ Universidade Paulista, Brasil \\ E-mail: irenilza.naas@docente.unip.br \\ Jair Minoro Abe \\ ORCID: https://orcid.org/0000-0003-2088-9065 \\ Universidade Paulista, Brasil \\ E-mail: jairabr@uol.com.br \\ Cristina Corrêa de Oliveira \\ ORCID: https://orcid.org/0000-0002-8629-6679 \\ Instituto Federal de São Paulo, Brasil \\ E-mail: crisolive@ifsp.edu.br \\ Luiz Roberto Forçan \\ ORCID: https://orcid.org/0000-0001-7376-830X \\ Universidade Paulista, Brasil \\ E-mail: luforcan@gmail.com
}

\section{Resumo}

A contratação de gestores na construção civil está paulatinamente mudando, sendo impostas pelas constantes mudanças de mercado. O objetivo deste estudo é desenvolver e avaliar a confiabilidade de um instrumento de avaliação de competências comportamentais para a contratação de gestores de projetos, minimizando os vieses subjetivos e possíveis conflitos dos avaliadores no processo seletivo. Pela necessidade de manipular dados incertos, conflitantes e mesmo falta de dados, o constructo utiliza a Lógica Paraconsistente Anotada Evidencial Ex, em duas dimensões, dotada de uma linguagem que pode expressar a evidência favorável e a evidência desfavorável das competências comportamentais, que tem a capacidade de abarcar as situações supracitadas. Utilizou-se o Índice de Validade de Conteúdo para a análise da confiabilidade, com uma escala Likert. As avaliações foram realizadas com seis juízes, sendo que na primeira etapa, três juízes ponderaram valores abaixo do esperado. Após explanação na segunda rodada, os juízes consideraram o instrumento viável, com escore de concordância $\boldsymbol{\mu} 86 \%$ e $\lambda 82 \%$ e Indice de Validade de Conteúdo com escore $\boldsymbol{\mu} 0,92$ e $\lambda$ 0,85, alcançando a verificação eficaz, e os procedimentos teóricos e analíticos são realizados e validados, sendo promissores na avaliação.

Palavras-chave: Competência; Instrumento de avaliação; Lógica paraconsistente anotada evidencial Ẽ

\begin{abstract}
The hiring of managers in civil construction is gradually changing, being imposed by constant market changes. This study aims to develop and evaluate an instrument's reliability for assessing behavioral competencies for hiring project managers, minimizing subjective bias and possible conflicts of evaluators in the selection process. Due to the need to manipulate uncertain, conflicting data and even lack of data, the construct uses the Paraconsistent Annotated Evidential Logic E $\boldsymbol{\tau}$, in two dimensions, endowed with a language that can express the favorable evidence and the unfavorable evidence of behavioral competencies, which can cover the situations mentioned above. The Content Validity Index was used to analyze the reliability using a Likert scale. The evaluations were carried out with six judges, and in the first stage, three judges weighed lower than expected values. After explanation in the second round, the judges considered the instrument viable, with an agreement score of $\boldsymbol{\mu} 86 \%$ and $\lambda 82 \%$ and Content Validity Index with a score of $\boldsymbol{\mu} 0.92$ and $\lambda 0.85$, achieving effective verification and procedures theoretical and analytical are carried out and validated, being promising in the evaluation.
\end{abstract}

Keywords: Competence; Evaluation instrument; Paraconsistent annotated evidential logic Ex. 


\begin{abstract}
Resumen
La contratación de gerentes en construcción civil está cambiando paulatinamente, siendo impuesta por los constantes cambios del mercado. El objetivo de este estudio es desarrollar y evaluar la confiabilidad de un instrumento de evaluación de competencias conductuales para la contratación de gerentes de proyecto, minimizando sesgos subjetivos y posibles conflictos de evaluadores en el proceso de selección. Debido a la necesidad de manipular datos inciertos, conflictivos e inclusa falta de datos, el constructo utiliza la Logica Paraconsistente Evidencial E $\boldsymbol{\tau}$, en dos dimensiones, dotada de un lenguaje que puede expresar evidencia favorable y evidencia desfavorable de competencias conductuales, que tiene la capacidad para cubrir las situaciones anteriores. Se utilizó el Índice de Validez de Contenido para analizar la confiabilidad, utilizando una escala Likert. Las evaluaciones se realizaron con seis jueces, y en la primera etapa, tres jueces pesaron valores inferiores a los esperados. Luego de la explicación en la segunda ronda, los jueces consideraron viable el instrumento, con un puntaje de acuerdo de $\mu$ $86 \%$ y $\lambda$ 82\% e Índice de Validez de Contenido con un puntaje de $\boldsymbol{\mu} 0.92$ y $\lambda \mathbf{\lambda} 0.85$, logrando verificación y procedimientos efectivos se llevan a cabo teóricos y analíticos. fuera y validado, siendo prometedor en la evaluación.
\end{abstract}

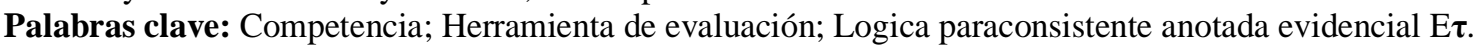

\title{
1. Introdução
}

O recrutamento e a seleção de gestor de projeto, dentro de uma organização, representam elevada importância, pois são profissionais que compõem o ativo principal e estratégico da empresa. Um equívoco nesse processo de contratação, compromete a produtividade do trabalho e o desempenho das equipes e, consequentemente, o desenvolvimento do projeto. Selecionar o profissional adequado, naturalmente é resultado de um processo de seleção eficaz, agrega valor para a missão, para os objetivos do projeto e na visão da empresa, pois as habilidades comportamentais do profissional, devem estar alinhados com os valores da empresa e da sociedade (Pells, 2021).

As vantagens na utilização da Lógica Paraconsistente Anotada Evidencial E $\tau$ (Lógica E $\tau$ ) (Abe, 2015), deve-se ao fato de que os parâmetros de entrada estão relacionados com a resposta do candidato, consolidando um instrumento de avaliação para a tomada de decisões, considerando o desempenho do candidato para uma contratação segura e estratégica. A avaliação da validade de conteúdo de um instrumento deve ser aplicada em todos os inventários, como por exemplo os de avaliação de candidatos, surveys, personalidades e testes educacionais (Alexandre \& Coluci, 2011), pois a literatura tem cada vez mais exigido uma correta avaliação das qualidades de instrumentos de apoio a decisão, tais como, confiabilidade (reliability), validade (validity), facilidade de uso (usability), sensibilidade (sensitivity) e responsividade (responsiveness).

Esta pesquisa teve como objetivo desenvolver um instrumento de avaliação de habilidades comportamentais adotando a Lógica Paraconsistente Anotada Evidencial E $\tau$ (Abe et al., 2011), na tomada de decisão, auxiliando a análise do candidato a gestor de projeto, com duas dimensões, sendo que cada uma é composta por nove questões, relacionadas com diferentes competências, utilizando a Lógica $\mathrm{E} \tau$ para mensurar o resultado da avaliação. O presente estudo apresenta os resultados da validade de conteúdo e de confiabilidade do Instrumento de Avaliação das Competências (IACC), concebido para avaliar as competências comportamentais de um candidato a gestor de projeto.

\section{Referencial Teórico}

Este tópico trata da fundamentação teórica das competências e suas relações com a gestão de projetos, assim como a aplicação conceitual na elaboração do instrumento de avaliação de competências.

\subsection{Competência}

A competência foi definida como uma motivação de desempenho e a capacidade de interagir com meio em que vive com eficácia (White, 1959) revisando a literatura, a competência para Fleury e Fleury (2011) é o conceito se refere à posse, por parte do profissional envolvido ou de uma empresa, e das características necessárias para se efetuar uma determinada atividade. A competência comportamental tem sido estudada e referenciada para uma nova modelagem ideal de profissionais qualificados, contribuindo para um ambiente em constante alteração em que os gestores de projetos atuam. Empregar gestores 
de projetos competente, no que lhe concerne, pode aumentar a satisfação no trabalho (Fonseca et al., 2013), melhorar a qualidade do serviço, e resultar no melhor desempenho financeiro nos projetos (Blayney et al., 2020).

O princípio da competência descreve conforme um compreender, realizar as atividades com responsabilidade e legalidade, que implica impulsionar, incorporar, transmitir conhecimentos, com ética e os recursos adquiridos, agregando de tal relevância economicamente à empresa, e a legitimidade social ao indivíduo. Acredita-se que o conceito evoluiu a partir da visão baseada em recursos, onde é denominado por diferentes nomes: competências organizacionais, competências distintas, capacidades distintas e dinâmicas, e as competências centrais (Enginoğlu \& Arikan, 2016), esses processos são considerados como uma visão de maturidade organizacional.

A capacidade de acumular, manter a utilização e desenvolver o mercado de produtos e serviços, decide as vantagens competitivas da corporação à longo prazo. A reserva de capacidade do gestor no contexto técnico é o que governa o escopo de gestão do projeto, para tanto, é determinado o perfil adequado para alto nível de gestão. Dentre as principais competências nos indivíduos descreve adaptar-se com as mudanças e situações questionáveis; pensar taticamente; tomar decisões com equilíbrio e exatidão diante das pressões de trabalho; aptidão para trabalhar em equipe; sabedoria para negociar quando houver contrariedade; proficiência para se sobressair de situações difíceis; diferenciar trabalho da vida pessoal; aperfeiçoamento de bom humor; e aptidão em ser adaptável e flexível.

Com base no estudo de Gramigna (2007), o domínio de algumas competências, faz grandes mudança no âmbito profissional, os novos talentos, mostram, dificuldades para atender as exigências e a demanda exigida pelo mundo globalizado. As competências essenciais e suas características individuais do modelo de competência, são desdobradas segundo orientações taxonômicas. As competências elencadas para este trabalho, foram selecionadas conforme representada na Tabela 1.

Tabela 1. Descrição de Competências.

\begin{tabular}{|c|c|}
\hline Competências & Descrição das competências \\
\hline $\begin{array}{l}\text { Adaptações e } \\
\text { Flexibilidade }\end{array}$ & $\begin{array}{l}\text { Capacitação em adaptar-se as diferenças exigidas do meio, considerando rever a } \\
\text { postura à frente de novas realidades. }\end{array}$ \\
\hline Empreender & $\begin{array}{l}\text { Facilidade na identificação de novas oportunidades e propor soluções inovadoras, } \\
\text { assertivas e adequadas ao contexto inserido. }\end{array}$ \\
\hline Negociação & $\begin{array}{l}\text { Capacidade de dialogar e resolver conflitos, atendendo satisfatoriamente a proposta } \\
\text { apresentada por ambas as partes. }\end{array}$ \\
\hline Trabalho em equipe & $\begin{array}{l}\text { Capacidade de criar esforço coletivo na resolução de problemas, considerando o } \\
\text { contexto inserido. }\end{array}$ \\
\hline Planejamento & $\begin{array}{l}\text { Capacidade de planejar e organizar ações, considerando os resultados tangíveis e } \\
\text { mensuráveis ao contexto inserido. }\end{array}$ \\
\hline Liderança & $\begin{array}{l}\text { Facilidade em motivar, influenciar e inspirar o grupo de pessoas para atingir os } \\
\text { melhores resultados ao contexto inserido. }\end{array}$ \\
\hline Comunicação & $\begin{array}{l}\text { Facilidade na troca de informações materializando o pensamento, sendo interlocutor } \\
\text { considerando o contexto inserido. }\end{array}$ \\
\hline Tomada de decisão & $\begin{array}{l}\text { Facilidade em solucionar e propor alternativas, garantindo o melhor resultado } \\
\text { tangível e mensurável ao contexto inserido. }\end{array}$ \\
\hline Resultados & $\begin{array}{l}\text { Capacidade e facilidade em atender aos objetivos e atingir as metas com os melhores } \\
\text { resultados ao contexto inserido. }\end{array}$ \\
\hline
\end{tabular}

Fonte: Adaptado de Gramigna (2007)

\subsection{Relação entre Competências e Gestores de Projetos}

Na competitividade do setor da indústria da construção, considerando as competências nas organizações (LoufraniFedida \& Saglietto, 2016), profissionais que não tem competência para competir, não tem capacidade para se manter num mercado em que as tendências de inovações e a qualidade de serviços, são principais requisitos para inovações no setor. As 
competências comportamentais, são de responsabilidade do gestor de projeto (Desmond, 2014), que conduz uma seleção de requisitos técnicos e comportamentais para um cliente, expondo a capacidade gerencial, e controlando todas as partes envolvidas do projeto. Ao mesmo tempo, algumas competências quando não utilizadas, podem diminuir a capacidade de gestão. Esse processo leva a uma rápida obsolescência do perfil de competências de um gestor de projetos (Różewski et al., 2013).

Para gerenciar com sucesso, são identificadas como um dos aspectos-chave com a combinação de elementos de competências de modo a produzir um resultado que deve ser alcançado e a maturidade de entendimento estratégico da organização. No estudo apresentado (Hayward, 2010) são destacadas as seguintes promissoras "competências essenciais", cuja relevância e seus desafios só irá aumentar no futuro, como a capacidade de adaptação, talento comercial e identificação de oportunidades de inovação, habilidades de comunicação para persuasão, e prestação de serviço de alto nível as partes interessadas (stakeholders). Segundo o PMI® (2020) o gestor de projetos é o responsável pelo resultado do projeto com impactos positivos ou negativos, o que denota a importância deste profissional nas organizações.

O que envolve o desenvolvimento de oportunidades de resolução de problemas e a tomada de decisão, bem como a responsabilidade pessoal (Belout \& Gauvreau, 2004) e empatia pelos colaboradores da empresa, que são as habilidades na construção de relacionamentos, complementa a dinâmica estrutural com a capacidade de motivar grupos, alcançando o sucesso em um ambiente heterogêneo. Portanto, é senso comum que as competências de um gestor de projeto é um fator-chave que influencia o resultado de um projeto (Liikamaa, 2015). Estudos mostram que as organizações que subestimam o gerenciamento de projetos como uma competência estratégica para disparar a mudança, relatam uma média de 67\% a mais de seus projetos que falharam inteiramente. Os líderes executivos identificaram como uma das mais importantes condições é garantir habilidades relevantes de gestores de projetos com (31\%), identificaram o fator para compreender o insucesso de gestão de projetos, sendo que o requisito é o abono de habilidades comportamentais, sendo considerado 58\% PMI® (2020).

\subsection{Entrevista por Competência}

A entrevista comportamental busca exemplos repetidos que produzem um padrão de comportamento e revelam as competências que o candidato possui, com o objetivo de prever o desempenho e o comportamento futuro (Latham et al., 1980). Infelizmente, os traços das competências comportamentais não aparecem necessariamente em um "curriculum vitae" ou declaração pessoal (Janz, 1982). Ao se falar da entrevista de seleção atualmente, refere-se a um dos campos da gestão de pessoa numa organização, com a tarefa de separar o candidato mais qualificado para referida função. Ancorada na visão de (Rabaglio, 2001), a seleção por competências pelo método Contexto, Ação e Resultado (CAR), as perguntas situacionais selecionadas por competência comportamental, propõe um suposto cenário ao candidato correlacionando a sua vivência em outros projetos, relacionando as perguntas com as situações do passado ou as lições aprendidas neste período e, sendo oportuno para o avaliado, compartilhar experiências que possam resultar numa compreensão mais abrangente. $\mathrm{O}$ modelo deste instrumento de avaliação conjunta, praticado entre avaliador e avaliado (candidato), é utilizada pelos dois lados simultaneamente, discutida e consensual. Neste método, há uma clareza e transparência na comunicação, exigindo da empresa uma cultura na avaliação com nível de maturidade elevada, principalmente na comunicação entre líderes e subordinados internamente.

O processo de seleção de gestores envolve fatores qualitativos, e a seleção é mais frequentemente atribuída a competências técnicas de gerenciamento de projetos (Sitohang et al., 2020), sendo aplicado com menos intensidade na avaliação quantitativa. A técnica da entrevista deste instrumento, é justamente propor uma análise das competências comportamentais pela Lógica $\mathrm{E} \tau$ com o propósito investigativo, qualificando o profissional que deverá demonstrar como seu diferencial competitivo, na gestão e os impactos em seus resultados como, por exemplo na: flexibilidade, tomada de decisão e 
os resultados alcançados. Alguns dos métodos convencionais como análise das habilidades descritas no documento "currículo vitae", avalia as aptidões sociais, comportamentais qualitativas, podendo levar a resultados de uma avaliação com parcialidade, sendo subjetiva e até mesmo incompleta, tornando o processo de seleção de gestores meramente formal com resultados incertos.

\subsection{Lógica Paraconsistente Anotada Evidencial E $\tau$}

A necessidade de se tomar decisão ocorre num momento de impasse em que a mais de uma opção. Cada um de nós toma decisões baseadas em aspectos intuitivos e subjetivos, e a subjetividade não tem medida perfeita ela é organizada, sistemática e objetiva, e a intuição é ação involuntária e inconsistente. A Lógica Paraconsistente Anotada Evidencial E $\tau$ figura entre as chamadas lógicas não clássicas por conter disposições contrárias a alguns dos princípios básicos da Lógica Aristotélica, tais como o princípio da contradição (Abe, 2015). Na visão aristotélica, qualquer afirmação é necessariamente verdadeira ou falsa. Segundo a Lógica Paraconsistente, uma sentença e a sua negação podem ser ambas verdadeiras.

A linguagem da Lógica $E \tau$ compõem-se de expressões onde p é uma proposição no sentido usual e o par $(\mu, \lambda)$ chamase constante de anotação. $\mu$ indica o grau de evidência favorável e $\lambda$ o grau de evidência desfavorável expressa por $p$, estando os valores de $\mu$ e $\lambda$ limitados entre 0 e 1 (Abe, 2015). Os estados da decisão abarcam quatro valores limites extremos: Os valores agrupam-se quando uma proposição é considerada "verdadeira" tomando a decisão positiva e a "falsa" decisão negativa. Apesar da Lógica $\mathrm{E} \tau$ abarcar doze estados lógicos, sendo quatro extremos, conforme Erro! Fonte de referência não encontrada., e oito não extremos, conforme pode ser constatado na Erro! Fonte de referência não encontrada., entretanto, não serão aplicados os estados não extremos neste estudo.

Figura 1. Representação do Reticulado $\tau$

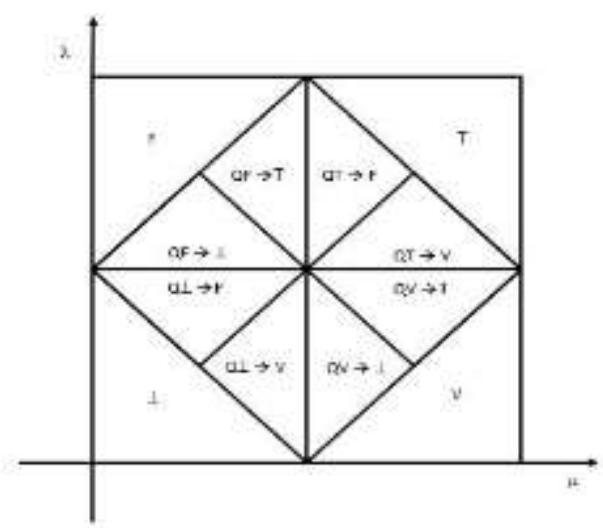

Tabela 2. Simbolização dos Estados Lógicos

\begin{tabular}{lc|lc}
\hline Extreme States & Symbol & Non-Extreme States & Symbol \\
\hline Verdadeiro & $\mathrm{V}$ & Quase-Verdade tendendo a Inconsistente & $\mathrm{Q}_{\mathrm{V}} \rightarrow \mathrm{T}$ \\
Falso & $\mathrm{F}$ & Quase-Verdade tendendo a Paracompleto & $\mathrm{Q}_{\mathrm{V}} \rightarrow \perp$ \\
Inconsistente & $\mathrm{T}$ & Quase-Falso tendendo a Inconsistente & $\mathrm{Q}_{\mathrm{F}} \rightarrow \mathrm{T}$ \\
Paracompleto & $\perp$ & Quase-Falso tendendo a Paracompleto & $\mathrm{Q}_{\mathrm{F}} \rightarrow \perp$ \\
& & Quase-Inconsistente tendendo a Verdadeiro & $\mathrm{Q}_{\mathrm{T}} \rightarrow \mathrm{V}$ \\
& & Quase-Inconsistente tendendo a Falso & $\mathrm{Q}_{\mathrm{T}} \rightarrow \mathrm{F}$ \\
& & Quase-Paracompleto tendendo a Verdadeiro & $\mathrm{Q}_{\perp} \rightarrow \mathrm{V}$ \\
& & Quase-Paracompleto tendendo a Falso & $\mathrm{Q}_{\perp} \rightarrow \mathrm{F}$ \\
\hline
\end{tabular}

Fonte: Abe (2015).

\subsection{Instrumentos de validação}

Instrumentos de avaliação empregam a escala Likert (Stevens, 1936) para avaliar a relevância e a representatividade; as respostas podem incluir na análise: $1=$ não relevante ou não representativo até o extremo de $4=$ item relevante ou representativo. Outros autores sugerem outras opções, sendo que o critério utilizado neste estudo consta na Tabela 6, com cinco valores escalares.

O procedimento de concordância, permite que dois ou mais avaliadores revisem e avaliem de forma independente a relevância de um conjunto de itens, em um instrumento de verificação. Apesar da literatura apresentar vários métodos, não há consenso entre os instrumentos de avaliação, devido à ausência de uma padronização rigorosa de avaliação (Alexandre \& Coluci, 2011). Outros autores propõem diferentes métodos para quantificar o grau de concordância. Dentre esses, destaca-se o grau de concordância, que é um método aplicado no cálculo da porcentagem de concordância inter-observadores (Alexandre \& 
Coluci, 2011) (Matos, 2014) aplicados conforme a Equação 1.

O Índice de Validade de Conteúdo (IVC) é calculado por meio da soma de concordância dos itens marcados por "3","4" e "5" pelos respondentes. Os itens que recebem a pontuação "1" ou "2" devem ser revisados. Dessa forma, o IVC tem sido também definido como "a proporção de itens que recebe uma pontuação de 3,4 e 5 pelos juízes.

Para verificar a validade de novos instrumentos, de uma forma geral, autores sugerem uma concordância mínima de 0,80 (Alexandre \& Coluci, 2011).

Equação 1 - \% de concordância

$$
\% \text { Concordância }=\frac{\text { Número de Juízes que concordam }}{\text { Número total de juízes }} \times 100
$$

$$
\begin{aligned}
& \text { Equação } 2 \text { - Índice de Validade de } \\
& \text { Conteúdo } \\
& \text { IVC }=\frac{\text { número de respostas } 4,5}{\text { número total de respostas }}
\end{aligned}
$$

Fonte: Adaptado de Alexandre (2011)

\section{Metodologia}

Para se atingir o objetivo proposto, foi utilizado o método quali-quantitativo (Pereira et al., 2018). Para avaliar o IACC, foi desenvolvido um questionário, utilizando a escala Likert (Vieira, 2009), qualificando cada questão quanto: a adequação (C1); a elaboração (C2); a objetividade (C3) e a compreensão semântica (C4).

A construção das perguntas baseou-se em critérios técnicos com as competências comportamentais e as mudanças de requisitos em projetos. As perguntas são abertas, permitindo uma elaboração mais detalhada dela, realizada pelo avaliador. As respostas do candidato são abertas, possibilitando um detalhamento de um cenário hipotético, ou mesmo de experiências anteriores. Os resultados das questões foram avaliados em duas dimensões, que analisam a evidência favorável e a evidência desfavorável na visão do avaliador, sendo que as respostas são compostas pelos graus de evidências, com valores pertencentes ao intervalo entre [0,1] (Abe, 2015). O método utilizado é apropriado para eliminar a subjetividade e os conflitos entre os avaliadores, de forma a distanciar os elementos de maior influência nas decisões.

O processo de seleção dos profissionais de gestão, aptos a participarem deste estudo, foram selecionados por apresentarem experiência e maturidade profissional, assim como conhecimento acadêmico sobre tais processos (Zikmund et al., 2010), pois eles desempenham o papel de especialistas, tais como diretores, gerentes e coordenadores em gerenciamento de projetos, agregando valor no processo de avaliação, contribuindo com o aperfeiçoamento do IACC.

A participação dos juízes se deu pela forma online, iniciando com a concordância de participação, de forma anônima, resguardando assim a privacidade dos juízes (especialistas), atendendo as normas vigentes e dentro da conformidade da lei. Existia a possibilidade dos juízes se identificarem apenas por e-mail.

O instrumento de avaliação derivou dos temas apresentados na fundamentação teórica, a fim de estabelecer as principais diretrizes de avaliação das competências comportamentais de um candidato ao cargo de gestão de projetos. O estudo foi desenvolvido em cinco etapas, sendo que a primeira envolveu um amplo estudo bibliográfico, incluindo periódicos, dos assuntos relacionados às competências comportamentais (Gramigna, 2007), especificamente nas bases de pesquisas internacionais e nacionais, nos últimos cinco anos, tais como: Institute of Electrical and Eletronics Engineers; American Psychological Association; American Physical Society; Emerald Insight; Science Direct; Web of Science; Conselho Federal de Psicologia e Directory of Open Access Journals. A estratégia de busca baseou-se nos descritores com a expressão booleana "E" - "competência" - "E" - "comportamental" - "E" - "gestão de projetos", e seus equivalente em inglês. Foram selecionadas as competências comportamentais (Gramigna, 2007), alinhadas com as competências organizacionais (Chipulu et al., 2013), especificamente para a indústria da construção civil. 


\subsection{Elementos do Constructo - Competência, Gestão de Mudanças e a Lógica E $\tau$}

As mudanças em projetos ocorrem quando as organizações buscam benefícios comerciais com a gestão de custos, com o aprimoramento dos serviços, agilidade e eficiência no suporte e nas estratégias de projeto. O objetivo desta etapa foi definir as questões que avaliam cada item da competência comportamental, conforme a Tabela 3 e Tabela 4 relacionando-as com o requisito de mudança. Foram definidas as duas dimensões para cada questão, sendo que primeira dimensão trata evidência favorável e a segunda dimensão trata a evidência desfavorável, conforme utilizado na Lógica E $\tau$. (Abe, 2015).

Por outro lado, houve a preocupação da objetividade, da clareza e do entendimento da questão associado a uma competência (Chipulu et al., 2013; Ng, 2018).

A estrutura beneficia todos os envolvidos na fase de entrevista ao candidato, sendo possível o aprimoramento da questão pelo avaliador durante esta etapa, explorando o conhecimento do candidato, apresentando cenários hipotéticos de gestão de projetos. A constância das perguntas foi baseada em fatos nos requisitos de mudança em projetos que, historicamente, o candidato poderá se apropriar e indicar suas experiências e as atribuições vivenciadas.

\subsection{O Instrumento de Avaliação de Competência Comportamental}

Estabelecida as duas dimensões do IACC do candidato, a primeira dimensão aborda as questões da evidência favorável, Q01 a Q09, conforme pode ser visto na Tabela 3, onde o avaliador quantifica a concordância com a resposta do candidato. A segunda dimensão, refere-se à evidência desfavorável, Q10 a Q18, conforme pode ser visto na Tabela 4, onde o avaliador quantifica a discordância dele com a resposta do candidato. Apesar das questões serem fechadas, aceitam respostas abertas, não restringindo a explanação do candidato (Utz, 2010), ou seja, considerando as experiências individuais de cada profissional, e juntamente, com seus históricos de decisões, relacionadas ao gerenciamento de projetos. 
Tabela 3. As questões de 1 a 9 se reportam às evidências favoráveis $(\mu)$.

\begin{tabular}{|c|c|c|}
\hline Questão & Descrição da questão & Critério \\
\hline Q1 & $\begin{array}{l}\text { Competência: Adaptação e Flexibilidade } \\
\text { P. Candidato: Caso o projeto tenha adaptações exigidas pelo contratante (cliente), } \\
\text { como você faria para se adaptar e flexibilizar as mudanças? } \\
\text { P. Avaliador: Quanto você acredita que o candidato foi convincente com a } \\
\text { flexibilização e adaptação apresentada? }\end{array}$ & \\
\hline Q2 & $\begin{array}{l}\text { Competência: Empreender } \\
\text { P. Candidato: Diante das mudanças solicitadas pelo contratante (cliente), qual a } \\
\text { solução imediata para apresentar de forma assertiva e adequada novas } \\
\text { oportunidades no projeto? } \\
\text { P. Avaliador: Quanto você acredita que o candidato foi convincente com a solução } \\
\text { apresentada? }\end{array}$ & \\
\hline Q3 & $\begin{array}{l}\text { Competência: Negociação } \\
\text { P. Candidato: Conforme solicitado pelo contratante (cliente) as mudanças no } \\
\text { projeto, qual melhor solução satisfatória para solucionar o problema sem ocasionar } \\
\text { conflitos? } \\
\text { P. Avaliador: Quanto você acredita que o candidato foi convincente com a solução } \\
\text { apresentada? }\end{array}$ & 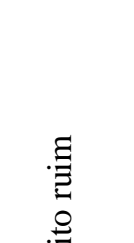 \\
\hline Q4 & $\begin{array}{l}\text { Competência: Trabalho em Equipe } \\
\text { P. Candidato: Diante das mudanças solicitadas pelo contratante (cliente), sua } \\
\text { equipe de projeto discorda das reais mudanças, qual o esforço coletivo para resolver } \\
\text { as incertezas de equipe? } \\
\text { P. Avaliador: Quanto você acredita que o candidato foi convincente com os } \\
\text { esforços e soluções apresentadas? }\end{array}$ & 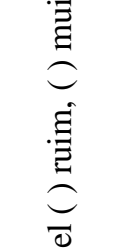 \\
\hline Q5 & $\begin{array}{l}\text { Competência: Planejamento } \\
\text { P. Candidato: Quando se trata de requisitos de projeto, as partes interessadas } \\
\text { firmam a data de início de projeto, qual a melhor estratégia para atingir os } \\
\text { resultados tangíveis e válidos? } \\
\text { P. Avaliador: Quanto você acredita que o candidato foi convincente com a solução } \\
\text { apresentada? }\end{array}$ & 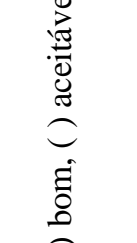 \\
\hline Q6 & $\begin{array}{l}\text { Competência: Liderança } \\
\text { P. Candidato: Explique o que é ser um líder e suas influências para atingir os } \\
\text { objetivos do gerenciamento de projeto? } \\
\text { P. Avaliador: Quanto você acredita que o candidato foi convincente com a solução } \\
\text { apresentada? }\end{array}$ & 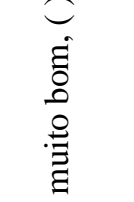 \\
\hline Q7 & $\begin{array}{l}\text { Competência: Comunicação } \\
\text { P. Candidato: Quais são as boas práticas e estratégias na argumentação das } \\
\text { mudanças solicitadas pelo contratante (cliente) e com a sua equipe de projeto? } \\
\text { P. Avaliador: Quanto você acredita que o candidato foi convincente com a solução } \\
\text { apresentada? }\end{array}$ & \\
\hline Q8 & $\begin{array}{l}\text { Competência: Tomada de decisão } \\
\text { P. Candidato: Nas mudanças solicitadas pelo contratante (cliente), qual a } \\
\text { capacidade de buscar e selecionar alternativas para garantir os melhores resultados } \\
\text { no projeto? } \\
\text { P. Avaliador: Quanto você acredita que o candidato foi convincente com as } \\
\text { decisões apresentadas? }\end{array}$ & \\
\hline Q9 & $\begin{array}{l}\text { Competência: Resultados } \\
\text { P. Candidato: Qual o principal risco nos resultados do projeto caso as mudanças } \\
\text { solicitadas pelo contratante (cliente) não sejam cumpridas? } \\
\text { P. Avaliador: Quanto você acredita que o candidato foi convincente com as } \\
\text { decisões e os resultados apresentados? }\end{array}$ & \\
\hline
\end{tabular}

Fonte: Adaptado de Gramigna (2007) 
Tabela 4. As questões de 10 a 18 se reportam às evidências desfavoráveis $(\lambda)$.

\begin{tabular}{|c|c|c|}
\hline Questão & Descrição da questão & Critério \\
\hline Q10 & $\begin{array}{l}\text { Competência: Adaptação e Flexibilidade } \\
\text { P. Candidato: O contratante (cliente) não aprovou as mudanças em projeto, } \\
\text { como você faria para flexibilizar e se adaptar as mudanças? } \\
\text { P. Avaliador: Quanto você desacredita que o candidato não foi convincente com } \\
\text { a flexibilização e adaptação apresentada? }\end{array}$ & \\
\hline Q11 & $\begin{array}{l}\text { Competência: Empreender } \\
\text { P. Candidato: Diante das mudanças solicitadas pelo contratante (cliente), quanto } \\
\text { você classificaria o insucesso das soluções apresentadas para o problema? } \\
\text { P. Avaliador: Quanto você acredita que o candidato foi convincente com a } \\
\text { solução apresentada? }\end{array}$ & \\
\hline Q12 & $\begin{array}{l}\text { Competência: Negociação } \\
\text { P. candidato: Diante das mudanças solicitadas pelo contratante (cliente), qual a } \\
\text { solução imediata para apresentar de forma assertiva e adequada novas } \\
\text { oportunidades no projeto? } \\
\text { P. Avaliador: Quanto você acredita que o candidato foi convincente com a } \\
\text { solução apresentada? }\end{array}$ & \\
\hline Q13 & $\begin{array}{l}\text { Competência: Trabalho em equipe } \\
\text { P. Candidato: Diante das mudanças solicitadas pelo contratante (cliente), sua } \\
\text { equipe de projeto discorda das reais mudanças, qual o esforço coletivo para } \\
\text { resolver as incertezas de equipe? } \\
\text { P. Avaliador: Quanto você desacredita nesta relação e esforço? }\end{array}$ & \\
\hline Q14 & $\begin{array}{l}\text { Competência: Planejamento } \\
\text { P. Candidato: Quando se trata de requisitos de projeto, as partes interessadas } \\
\text { firmam a data de início de projeto, qual a melhor estratégia para atingir os } \\
\text { resultados tangíveis e válidos? } \\
\text { P. Avaliador: Quanto você classificaria o insucesso das soluções apresentadas } \\
\text { para o problema? }\end{array}$ & \\
\hline Q15 & $\begin{array}{l}\text { Competência: Liderança } \\
\text { P. Candidato: Explique o que é ser um líder e suas influências para atingir os } \\
\text { objetivos do gerenciamento de projeto? } \\
\text { P. Avaliador: Quanto você classificaria considerando que o candidato não tem } \\
\text { potencial de liderança }\end{array}$ & : \\
\hline Q16 & $\begin{array}{l}\text { Competência: Comunicação } \\
\text { P. candidato: Quais são as boas práticas e estratégias na argumentação das } \\
\text { mudanças solicitadas pelo contratante(cliente) à sua equipe de projeto? } \\
\text { P. Avaliador: Quanto você desacredita que o candidato apresentou uma solução } \\
\text { convincente? }\end{array}$ & 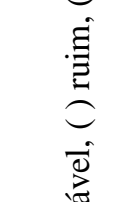 \\
\hline Q17 & $\begin{array}{l}\text { Competência: Tomada de decisão } \\
\text { P. Candidato: Nas mudanças solicitadas pelo contratante (cliente), qual a } \\
\text { capacidade de buscar e selecionar alternativas, identificando a que garantam o } \\
\text { melhor resultado no projeto? } \\
\text { P. Avaliador: Quanto você acredita que a apresentação não foi convincente com } \\
\text { as soluções e as decisões? }\end{array}$ & 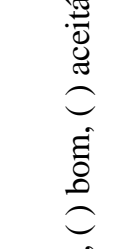 \\
\hline Q18 & $\begin{array}{l}\text { Competência: Resultados } \\
\text { P. Candidato: Qual o principal risco nos resultados do projeto caso as mudanças } \\
\text { solicitadas pelo contratante (cliente) não sejam cumpridas? } \\
\text { P. Avaliador: Quanto você acredita que o candidato não foi convincente com as } \\
\text { decisões e os resultados apresentados }\end{array}$ & 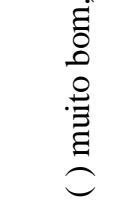 \\
\hline
\end{tabular}

Fonte: Adaptado de Gramigna (2007)

\subsection{Juízes (Respondentes)}

Foram selecionados vinte profissionais especialistas em gestão de projetos, como população-alvo, para avaliação do estágio inicial. Destes, apenas seis respondentes participaram da primeira etapa, definida como população amostrada. Os outros quatorze respondentes, participarão apenas da segunda etapa do estudo, para não haver interferência na aplicação do instrumento ou por não apresentarem o mesmo nível de maturidade profissional dos elegíveis. 
Foi adotado um processo sistemático de avaliação sendo dada uma atenção especial na ambientação dos respondentes iniciando com orientações sobre o instrumento e a Lógica $\mathrm{E} \tau$. Fez-se necessário, em uma avaliação de instrumento, apresentar a base conceitual do constructo, bem como de todas as etapas para o processo de avaliação (Grant, 1997). A Tabela 5 descreve os cargos dos juízes:

Tabela 5. Descrição de cargo dos Juízes Elegíveis.

\begin{tabular}{l|l}
\hline JUÍZES & \multicolumn{1}{c}{ CARGO } \\
\hline J1 & Diretor Administrativo de Novos Negócios e Projetos \\
J2 & Gerente de Planejamento e Gestão de Empreendimentos \\
J3 & Diretor de Projetos \\
J4 & Diretor Técnico de Projetos \\
J5 & Diretor de Tecnologia \\
J6 & Coordenador Geral de Projetos \\
\hline
\end{tabular}

Fonte: Autores.

\subsection{A avaliação do IACC}

O IACC foi avaliado utilizando a escala Likert, com os valores qualitativos, que foram normalizados para valores quantitativos. As opiniões dos respondentes variam, conforme as diferenças nos pensamentos distintos, na subjetividade ou na incerteza da resposta. Utilizou-se o método de distribuição escalar, com valores, variando de zero a um, nos extremos entre: "muito bom" até "muito ruim", correspondendo os critérios numéricos atribuído ao IVC, conforme pode ser conferido na Tabela 6. As questões foram avaliadas segundo os critérios de: (C1) adequação, (C2) elaboração, (C3) objetividade e (C4) compreensão. A escolha das respostas tem o compromisso de considerar a fidedignidade dos respondentes.

Tabela 6. Critério de Avaliação utilizando a escala adaptada de Likert.

\begin{tabular}{lll}
\hline $\mathbf{N}^{\mathbf{0}}$ & $\begin{array}{l}\text { Critério } \\
\text { Qualitativo }\end{array}$ & $\begin{array}{l}\text { Valores } \\
\text { Escalar }\end{array}$ \\
\hline 1 & Muito Ruim & 0,00 \\
2 & Ruim & 0,25 \\
3 & Aceitável & 0,50 \\
4 & Bom & 0,75 \\
5 & Muito Bom & 1,00 \\
\hline
\end{tabular}

Fonte: Autores.

\subsection{Etapa de avaliação dos juízes}

Realizou-se duas rodadas de avaliação do instrumento, sendo que a primeira rodada utilizou um formulário eletrônico desenvolvido com a ferramenta Microsoft-Forms ${ }^{\circledR}$, dividindo as questões em duas dimensões conforme descreve no item 3.2. O instrumento foi avaliado pela população elegível, no período de 20/10/2020 a 03/11/2020, e a duração média das avaliações foi de 20 minutos. Foi realizada uma entrevista individual com três juízes, e após explanação da lógica, foi dado início a segunda rodada, seguindo todas as etapas da primeira rodada.

\section{Resultados e Discussão}

As respostas foram analisadas mediante a comparação de seis juízes definidos para validação do IACC. A partir da normalização dos dados, atendendo as instruções da Tabela 6, Tabela 8 - Total de IVC por Juízes, considerando apenas a pontuação 4 e 5, conforme a Equação 2.

Os resultados da concordância para $(\mu, \lambda)$ demonstram que três juízes (J4, J5 e J6) não concordaram com a primeira 
rodada de questões, conforme destacado na Tabela 7, avaliando abaixo do satisfatório, com o resultado de $\mu=72 \%$ e $\lambda=67 \%$. A literatura indica que valores abaixo de $80 \%$ devem ser avaliados e reestruturados, exigindo uma nova avaliação (Alexandre \& Coluci, 2011).

Os valores da segunda rodada, que se encontram na parte inferior da, apresentam uma melhoria na concordância dos juízes, com o índice de $\mu=86 \%$ e $\lambda=82 \%$.

Tabela 7. \% Concordância Total por Juízes.

\begin{tabular}{ccccccccc}
\hline & & $\mathbf{J 1}$ & $\mathbf{J 2}$ & $\mathbf{J 3}$ & $\mathbf{J 4}$ & $\mathbf{J 5}$ & $\mathbf{J 6}$ & Média de Concordância \\
\hline \multirow{3}{*}{$\mathbf{1}^{\mathbf{a}}$ Rodada } & $\boldsymbol{\mu}$ & $94 \%$ & $81 \%$ & $92 \%$ & $\mathbf{8 8 \%}$ & $\mathbf{3 8 \%}$ & $\mathbf{4 1 \%}$ & $72 \%$ \\
& $\lambda$ & $97 \%$ & $89 \%$ & $79 \%$ & $\mathbf{6 0 \%}$ & $\mathbf{3 2 \%}$ & $\mathbf{4 5 \%}$ & $67 \%$ \\
& $\boldsymbol{\mu}$ & $94 \%$ & $81 \%$ & $92 \%$ & $\mathbf{8 8 \%}$ & $\mathbf{8 3 \%}$ & $\mathbf{7 9 \%}$ & $\mathbf{8 6 \%}$ \\
$\mathbf{2}^{\mathbf{a}}$ Rodada & $\lambda$ & $97 \%$ & $89 \%$ & $79 \%$ & $\mathbf{7 2 \%}$ & $\mathbf{7 6 \%}$ & $\mathbf{7 8 \%}$ & $\mathbf{8 2 \%}$ \\
\hline
\end{tabular}

Fonte: Autores.

A análise do cálculo de IVC, na primeira rodada, apresenta resultados satisfatórios para dimensão $(\mu)$ e valores insatisfatórios para a dimensão $(\lambda)$ conforme a Tabela 8. Após a segunda rodada, o resultado da dimensão $(\lambda)$ apresentou uma melhoria, sucedendo a etapa metodológica da explanação com os juízes, com valor acima de 0,75 do total de respostas. $\mathrm{O}$ estudo mostra que houve um resultado acima de 0,80 na média de concordância da dimensão $(\lambda)$.

Tabela 8. Total de IVC por Juízes.

\begin{tabular}{ccccccccc}
\hline & & J1 & J2 & J3 & J4 & J5 & J6 & Média de IVC \\
\hline \multirow{3}{*}{$1^{\text {a Rodada }}$} & $\boldsymbol{\mu}$ & 1,00 & 0,92 & 0,97 & 0,89 & 0,92 & 0,80 & 0,92 \\
& $\boldsymbol{\lambda}$ & 1,00 & 1,00 & 0,81 & $\mathbf{0 , 2 2}$ & $\mathbf{0 , 0 0}$ & $\mathbf{0 , 1 1}$ & $\mathbf{0 , 5 2}$ \\
$2^{\text {a }}$ Rodada & $\boldsymbol{\mu}$ & 1,00 & 0,92 & 0,97 & 0,89 & 0,92 & 0,80 & 0,92 \\
& $\boldsymbol{\lambda}$ & 1,00 & 1,00 & 0,81 & $\mathbf{0 , 6 7}$ & $\mathbf{0 , 8 9}$ & $\mathbf{0 , 7 2}$ & $\mathbf{0 , 8 5}$ \\
\hline
\end{tabular}

Fonte: Autores.

Apesar dos juízes-respondentes estarem mais habituados a questões qualitativas, onde o eles escolhem o ponto que melhor expressa seus entendimentos em relação as variáveis que estão sendo avaliadas; o uso da Lógica $\mathrm{E} \tau$ pode dificultar o julgamento da questão, em virtude do entendimento das duas dimensões, evidência favorável e evidência desfavorável. A primeira rodada do estudo evidenciou a falta de concordância entre os juízes, resultando em uma apreciação e reelaboração das questões, por parte dos autores, após a primeira rodada; exigindo uma reavaliação das questões pelos juízes, considerando a exposição da Lógica $\mathrm{E} \tau$ e suas aplicações.

A indústria da construção civil, que se caracteriza em desenvolver e executar projetos, ou bens tangíveis, necessita de execução e gerenciamento de projetos conforme especificação de escopo. Os desafios do mercado da construção civil e as incertezas no gerenciamento de projeto, pressionaram as empresas a se adaptarem aos novos modelos de gestão. Os projetos gerenciados por meio da aplicação de modelos preditivos ou adaptativos pode transformar uma organização, seja por meio do desenvolvimento de novos produtos e conceitos ou pelo aprimoramento dos processos seletivos.

O IACC se propõe a avaliar as competências comportamentais do candidato a gestor de projeto, de uma forma bidimensional, aplicando a Lógica $\mathrm{E} \tau$, com questões que aferem a evidência favorável e a evidência desfavorável, nos diferentes aspectos relacionados com as experiências anteriores do candidato, minimizando assim, a subjetividade e os conflitos entre os avaliadores. Os instrumentos de avaliações de competências, que mensuram a competência do candidato, auxiliam na tomada de decisão dos gestores, considerando o desempenho do candidato para uma contratação segura e estratégica.

Ressalta-se que este estudo faz parte da etapa inicial do processo de desenvolvimento e análise do IACC, dado que a 
próxima etapa consistirá na aplicação do mesmo com o público-alvo, ou seja, gestores de projetos, avaliando uma persona a fim de averiguar a confiabilidade do instrumento. Compreender a aplicação de um instrumento de avaliação, é essencial para os profissionais da área de recrutamento e seleção e de gerenciamento de projetos, tendo em vista o atual cenário de avaliações qualitativas, que proporciona cada vez mais resultados confiáveis e fidedignos para uma contratação de gestores com eficiência no gerenciamento de projetos.

\section{Conclusão}

Foi desenvolvido um modelo de avaliação de projetos utilizando o método da lógica paraconsistente. Na primeira rodada do processo de avaliação do IACC, não se obteve concordância unânimes entre os avaliadores, provavelmente por desconhecerem a Lógica $\mathrm{E} \tau$. Uma etapa essencial, foi o alinhamento dos conceitos desta lógica, provendo embasamento teórico para a segunda rodada. A avaliação crítica dos juízes propiciou o aprimoramento do IACC, resumidamente na sua forma e relevantemente no seu conteúdo, resultando em um instrumento mais adaptado para o uso dos avaliadores.

O percentual de concordância na segunda rodada para foi superior a $80 \%$ nas duas dimensões, conforme indica a literatura. Este percentual foi decidido em consequência da quantidade de respondentes elegíveis no processo de avaliação, uma vez que a literatura recomenda no mínimo cinco juízes para atingir uma taxa de $90 \%$.

\section{Referências}

Abe, J. M. (2015). Paraconsistent intelligent-based systems: New trends in the applications of paraconsistency (Vol. 94). Springer.

Abe, J. M., Da Silva Filho, J. I., Celestino, U., \& Corrêa, A. H. (2011). Lógica Paraconsistente Anotada Evidencial Et. (Comunicar, Ed.)

Alexandre, N. M., \& Coluci, M. Z. (2011). Validade de conteúdo nos processos de construção e adaptação de instrumentos de medidas. Ciência \& Saúde Coletiva, 16, 3061-3068. 10.1590/s1413-81232011000800006

Belout, A., \& Gauvreau, C. (2004). Factors influencing project success: the impact of human resource management. International journal of project management, 22, 1-11.

Blayney, C., Cormier-MacBurnie, P., \& Young, J. D. (2020). The devolution of human resource management to line managers: A preliminary examination of the hotel industry in Halifax, Canada. Journal of Human Resources in Hospitality \& Tourism, 19, 443-472. 10.1080/15332845.2020.1763760

Chipulu, M., Neoh, J. G., Ojiako, U., \& Williams, T. (2013). A Multidimensional Analysis of Project Manager Competences. IEEE Transactions on Engineering Management, 60, 506-517. 10.1109/tem.2012.2215330

Desmond, C. (2014). Multiple project managers. IEEE Engineering Management Review, 42, 12-12. 10.1109/emr.2014.2314543

Enginoğlu, D., \& Arikan, C. L. (2016). A literature review on core competencies. International Journal of Management (IJM), 7.

Fleury, A., \& Fleury, M. T. (2011). Brazilian multinationals: competences for internationalization. Cambridge University Press.

Fonseca, I. S., Araújo, T. M., Bernardes, K. O., \& Amado, N. (2013). Apoio social e satisfação no trabalho em funcionários de uma empresa de petróleo. Psicología para América Latina, 43-56.

Gramigna, M. R. (2007). Modelo de competências e gestão dos talentos. Em Modelo de competências e gestão dos talentos (pp. 24 1-241).

Grant, L. L. (1997). Seleção e uso de especialistas em conteúdo para desenvolvimento de instrumentos. (W. O. Library, Ed.) 20.

Hayward, S. (4 de 2010). Engaging employees through whole leadership. Strategic HR Review, 9, 11-17. 10.1108/14754391011040028

Janz, T. (1982). Comparações iniciais de entrevistas de descrição de comportamento padronizado versus entrevistas não estruturadas. Comparações iniciais de entrevistas de descrição de comportamento padronizado versus entrevistas não estruturadas, 67.

Latham, G. P., Saari, L. M., Pursell, E. D., \& Campion, M. A. (1980). The situational interview. Journal of Applied Psychology, 65, 422-427. 10.1037/00219010.65 .4 .422

Liikamaa, K. (2015). Developing Project Managerltextquotesingles Competencies: A Collective View of the Most Important Competencies. Procedia Manufacturing, 3, 681-687. 10.1016/j.promfg.2015.07.305

Loufrani-Fedida, S., \& Saglietto, L. (2016). Mechanisms for Managing Competencies in Project-Based Organizations: An Integrative Multilevel Analysis. Long Range Planning, 49, 72-89. 10.1016/j.lrp.2014.09.001

Matos, D. A. (2014). Confiabilidade e concordância entre juízes: aplicações na área educacional. Repositório Institucional Universidade Federal de Ouro Preto. http://dx.doi.org/10.18222/eae255920142750 
Research, Society and Development, v. 10, n. 4, e7610413444, 2021

(CC BY 4.0) | ISSN 2525-3409 | DOI: http://dx.doi.org/10.33448/rsd-v10i4.13444

Ng, J. J. (2018). A deeper look into a project management tool: The Change Register. IEEE Engineering Management Review, 46, 24-26. $10.1109 / \mathrm{emr} .2018 .2809921$

Pells, D. L. (2021). Project Management needs a Higher Purpose. Project Management needs a Higher Purpose, Vol. X, Issue I. (P. W. Journal, Ed.) Retrieved from https://pmworldlibrary.net/wp-content/uploads/2021/01/pmwj101-Jan2021-Pells-project-management-needs-a-higher-purpose-part-1-2.pdf

Pereira, A. S., Shitsuka, D. M., Parreira, F. J., \& Shitsuka, R. (2018). Metodologia da pesquisa cientifica. Fonte: http://repositorio.ufsm.br/handle/1/15824

Project Management Institute. (2 de 2020). Ahead of the CurveForging a Future-Focused Culture. Ahead of the CurveForging a Future-Focused Culture. Pensilvânia, Condado de Delaware, USA. Fonte: https://www.pmi.org/learning/library/forging-future-focused-culture-11908

Rabaglio, M. O. (2001). Seleção por competências. (2).

Różewski, P., Małachowski, B., Jankowski, J., Prys, M., \& Dańczura, P. (2013). Preliminares para a construção de sistema de gestão de competência dinâmica. 2013 Conferência Federada sobre Ciência da Computação e Sistemas de Informação.

Sitohang, Y. F., Pratami, D., \& Bay, A. F. (2020). Competency Evaluation of Project Manager Performance in Network Construction Projects. 2020 Fifth International Conference on Informatics and Computing (ICIC). IEEE. 10.1109/icic50835.2020.9288580

Stevens, S. S. (1936). A scale for the measurement of a psychological magnitude: loudness. Psychological Review, 43, 405-416. 10.1037/h0058773

Utz, K. (2010). A Subjetividade na "Ciência da Lógica". Veritas, 55.

Vieira, S. (2009). Como elaborar questionários. Atlas.

White, R. W. (1959). Motivation reconsidered the concept of competence. Psychological Review, 66, 297-333. 10.1037/h0040934

Zikmund, W. G., Babin, B. J., Carr, J. C., \& Griffin, M. (2010). Business Research Methods (Ninth Edition ed.). (C. Learning, Ed.) South-Western College Pub. 